\title{
Study of the Performance of Graded Palm Kernel Shells (PKS) As Partial Replacement for Coarse Aggregates in Hot Mix Asphalt (HMA) Binder Course with Zycotherm Chemical Additive
}

\author{
Olutaiwo, A. O.1 and Nnoka, C.C.2 \\ ${ }^{1,2}$ Department of Civil and Environmental Engineering, University Of Lagos, Nigeria
}

\begin{abstract}
The study looked at the potentials of palm kernel shells as coarse aggregates in an asphalt binder course using bitumen modified with zycotherm chemical additive. It also tries to investigate the effects of the modifier on asphalt concrete. All the volumetric and physical properties of the asphalt mixtures were evaluated using Marshall Mix design procedures. PKS percentage rates used were 0\%, 25\%, 50\%, 75\% and 100\% by weight of the total coarse aggregate size at $4-8 \mathrm{~mm}$. Seventy five (75) test samples of compacted asphalt mix were prepared, 15 samples for the control mix and 60 samples for the PKS portions. The samples were prepared by varying bitumen contents from $4.5 \%-6.5 \%$ of the weight of the total aggregate weights used. The observed results show that at optimum binder contents $(O B C)$, with zycotherm chemical additive, $75 \%$ and $100 \%$ replacement could be used for light - heavily Trafficked roads. However, 25\% and 50\% replacements could be used for light traffic situations as VFB values observed at optimum binder contents were slightly above recommended limits. From the results observed, it could be established that PKS is a viable agricultural waste product that could be used in road constructions as replacement of coarse aggregate in binder courses. When comparisons were made with asphalt concretes produced with the modified bitumen and plain bitumen, results showed that the zycotherm chemical additive positively affects asphalt concrete as improvements were seen in volumetric and physical properties. The result comparisons showed Marshall Stability, flow and void characteristics (Va, VMA, and VFB) were all improved as bitumen content required was lowered for the same mix design proportion and compaction load compared. This could be cost effective on the long run.
\end{abstract}

Keywords: Marshall Mix Design, Palm Kernel Shells, Binder Course, Coarse Aggregates, Zycotherm Chemical Additive.

\section{INTRODUCTION}

As developing nations worldwide strive for economic growth, sustainability and stability as well as to close the gap on the developed nations in terms of infrastructural innovations and development, efforts should be geared towards innovative developments and eco-friendly and economically viable ideas. One of such ideas is the re-use of by-product of domestic, mining and industrial activities. Recent developments in technologies and researches have continued to prove that there are several successful ways to make use of these so called wastes or by-products. One of these solutions is to use them in road construction as aggregates to replace or partially replace conventional materials. In the same light, the practice of modifying asphalt binders have been thought to offer improved performances over conventional binders and as such improve asphaltic mixtures for better performances of asphaltic paving surfaces.

The road construction industry depends majorly on conventional materials such as asphalt cement, granite, sand and fillers for the production of asphalt concrete. The costly nature of these materials have greatly hindered the course of development of road pavements in the country, herein lies the need for engineering considerations on available cheaper materials to invariably reduce construction costs for growth and development. Research studies in material sciences and engineering continually prove the potentials of having local materials to partially and fully replace these costly conventional ones. Historically, agricultural and industrial wastes have created waste management and pollution problems. However the use of agricultural wastes to complement other traditional materials in construction provides both practical and economic advantages.

Research studies have been thoroughly carried out using PKS; these include studies and investigations in soil compaction and stabilization, to using PKS as a viable source of coarse aggregate in structural concrete mostly in the production of light weight concrete and a few studies on its use also as aggregates (coarse and fines) in bituminous or asphalt concrete works. Researches were also conducted on the viability of crushed PKS as fine aggregates. Mohammed et al (2014), carried out research were to this effect. They studied the potential of crushed palm kernel shells as partial replacements of fine aggregate in asphalt concrete. The results obtained 
showed that the samples with 10 and 50\% partial replacement of fine aggregate (sand) with crushed palm kernel shell were within the specifications for asphalt concrete roads.

In investigative studies carried out by Olutaiwo and Owolabi (2015) on the effects of partial replacement of coarse aggregate with graded palm kernel shells in asphalt binder course at varying percentages of PKS content rates by weight of total coarse aggregate size of 4- 8mm. Marshall tests conducted established that PKS was a viable agricultural waste product that could be used as coarse aggregate at a specific percentage in the production of asphaltic binder courses for light to medium trafficked roads. Ndoke (2006) also investigated the potential of palm kernel shells as coarse aggregate in road binder course with emphasis on strength of the asphalt concrete as given by the Marshall Stability and flow values. In the study, it was observed that palm kernel shells could be used to replace coarse aggregate up to $10 \%$ for heavily Trafficked roads and $100 \%$ replacement was possible for light trafficked roads in rural areas.

Modifying bituminous materials can bring real benefits to highway maintenance and construction, in terms of better and long lasting roads and savings in total road life [Mangesh et al (2012)]. Harish and Shirakumar (2013) investigated the effects of modifying bituminous concrete with crumb rubber and waste shredded thermo-plastics and significant improvements in properties like Marshall stability, retained stability, indirect tensile strength where observed in comparison with conventional mix. Bamidele et al (2013) in their investigative research study on the use of shredded PWS (pure water sachets) to modify bitumen at various percentages weight of bitumen observed increases in viscosity decreasing penetration with subsequent increase in PWS while the values of the softening point increased with respect to increase in PWS. Ashik Bellary and Lokesh Gupta (2016) in there research publication on the use of GGBS as filler and zycotherm as chemical additive $(0.1 \%$ weight of asphalt/bitumen) for a bituminous concrete mixture observed satisfactory test results as regards standard MORT\&H specifications which is in line with asphalt institute standards. Likewise, Rohith $n$ and J.Ranjitha (2013) on their study publication on the Marshall Stability properties of warm mix asphalt using zycotherm as a chemical additive also concluded that stability \& Marshall Properties were improved for the wma mix by the addition of the additive.

\section{MATERIALS AND METHODS}

The typical materials which constituted the asphalt concrete where stone dust (0-5 mm), river sand (0-4 $\mathrm{mm}$ ) and crushed stone of size ranges of 4-8 mm, 8-16 mm and 16-24 mm. These where all obtained from Julius Berger Construction Company Stockpiles at Apapa in Lagos state Nigeria. The palm kernel shells used in the aggregate replacement were obtained locally from Badagry market also in Lagos state south west of Nigeria. Bitumen of 60/70 penetration grade modified with zycotherm chemical additive was used in the test study. This was used to produce the entire test specimens. The bitumen was supplied by Julius Berger construction company which was modified with zycotherm chemical additive supplied by VXL limited (Zycosoil) Ilupeju Lagos state specified percentage proportions of the samples used for combined gradation in the production of the asphalt concrete for the binder course was carried out as follows: $35 \%$ stone dust $(0-5 \mathrm{~mm}), 7 \%$ river sand $(0-4 \mathrm{~mm})$, $10 \%$ crushed stone (size $4-8 \mathrm{~mm}$ ), 15\% crushed stone (size 8-16 mm) and 33\% crushed stone (size 16-24 mm) with bitumen content between $4.5 \%$ and $6.5 \%$ at varying increments of $0.5 \%$. The PKS aggregate samples were varied between $25 \%, 50 \%, 75 \%$ and $100 \%$ partial to full replacement of coarse aggregate (size $4-8 \mathrm{~mm}$ ).

Blending was done at a temperature of $145-160^{\circ} \mathrm{c}$ and allowed to reduce to a temperature of $145^{\circ} \mathrm{c}$ before it was compacted on the both sides with 50 blows to obtain cylindrical samples. Marshall Stability and flow tests were carried out at a temperature of $60^{\circ} \mathrm{c}$ on the samples. Other tests carried out include specific gravity tests, asphalt content determination by solvent extraction and aggregate gradation tests. Preliminary tests were carried out on both the aggregates to be used as well as the zycotherm modified bitumen used. Test results could be seen in the tables below.

Table 1: Mix Proportion in Percent with Specific Gravities of the Aggregates Used

\begin{tabular}{|l|l|l|}
\hline Aggregate Sizes Used & Mix Proportion (\%) & Specific Gravity \\
\hline $16 \mathrm{~mm}-24 \mathrm{~mm}$ Crushed Stone & 33 & 2.760 \\
\hline $8 \mathrm{~mm}-16 \mathrm{~mm}$ Crushed Stone & 15 & 2.677 \\
\hline $4 \mathrm{~mm}-8 \mathrm{~mm}$ Crushed Stone & 10 & 2.670 \\
\hline $0-5 \mathrm{~mm}$ Stone Dust & 35 & 2.600 \\
\hline $0-4 \mathrm{~mm}$ River Sand & 7 & 2.665 \\
\hline PKS & VARIED & 1.620 \\
\hline
\end{tabular}

Courtesy: Julius Berger Company Asphalt Labs

Table 2: Some Properties of the Modified Bitumen Used

\begin{tabular}{|l|l|l|}
\hline Asphalt Property & Test Results & Specifications \\
\hline Specific Gravity At $25^{\circ} \mathrm{c}$ & 1.04 & $\geq 1$ \\
\hline Softening Point (Ball \& Ring) ${ }^{\circ} \mathrm{c}$ & 51 & $>50^{\circ} \mathrm{c}$ \\
\hline Penetration At $25^{0} \mathrm{c}-0.1 \mathrm{~mm}$ & 66 & $60-70$ \\
\hline Solubility In Trichloroethylene \% By Weight & 99 & 99 \\
\hline
\end{tabular}




\section{TEST RESULTS AND DISCUSSION}

3.1 Aggregate Gradation Analysis

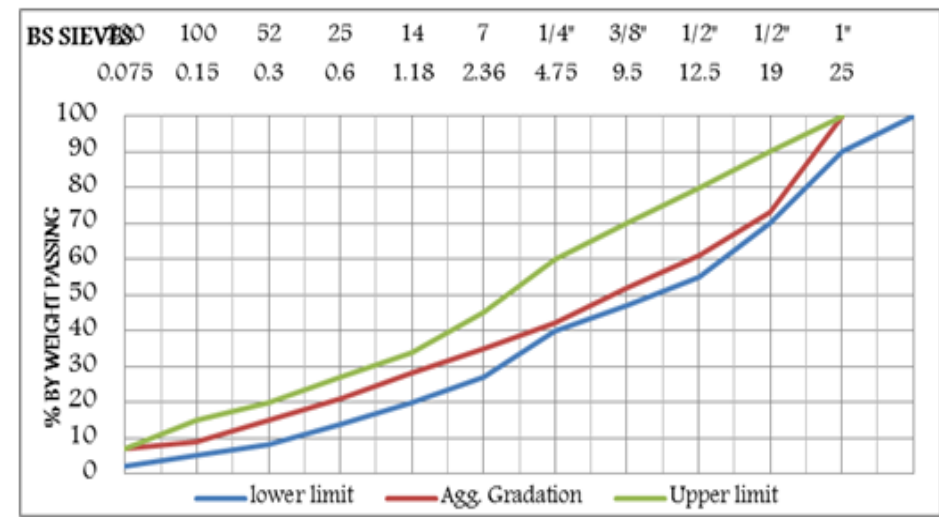

\begin{tabular}{|l|l|}
\hline Sieve & $\%$ \\
\hline BS & Passing \\
\hline 31.75 & - \\
\hline 25 & 100 \\
\hline 19 & 73 \\
\hline 12.5 & 61 \\
\hline 9.5 & 52 \\
\hline 4.75 & 42 \\
\hline 2.36 & 35 \\
\hline 1.18 & 28 \\
\hline 0.6 & 21 \\
\hline 0.3 & 15 \\
\hline 0.15 & 9 \\
\hline 0.075 & 7 \\
\hline
\end{tabular}

Fig.1: Sample Of Binder Course Aggregate Gradation Envelope 5.5\% Binder Content (75\%PKS) Note: Other Aggregate Gradations At 0\%, 25\%, 75\% And 100\% PKS Replacement Rates Were Derived and All Were Within the Gradation Envelope According To Binder Course Requirements of FMWH General Specification for Roads and Bridges (1994).

\subsection{Marshall Test Results and Analysis}

Table 3: Marshall Properties of Hot Mix Asphalt Prepared Using Zycotherm And 0\% PKS Replacement as Coarse Aggregate Material (4-8mm)

\begin{tabular}{|l|l|l|l|l|l|l|}
\hline $\begin{array}{l}\text { Binder } \\
\text { Content }(\%)\end{array}$ & $\begin{array}{l}\text { Unit Weight } \\
(\mathrm{g} / \mathrm{ml})\end{array}$ & $\begin{array}{l}\text { Marshall } \\
\text { Stability }(\mathrm{KN})\end{array}$ & $\begin{array}{l}\text { Flow } \\
(\mathrm{mm})\end{array}$ & $\begin{array}{l}\text { Total Air Voids } \\
(\mathrm{Va})(\%)\end{array}$ & $\begin{array}{l}\text { Voids Filled With } \\
\text { Bitumen (VFB) (\%) }\end{array}$ & $\begin{array}{l}\text { Voids In Mineral } \\
\text { Aggregate }(\text { VMA) }(\%)\end{array}$ \\
\hline 4.5 & 2.293 & 12.83 & 3.53 & 5.40 & 64.75 & 15.32 \\
\hline 5.0 & 2.301 & 13.97 & 3.73 & 4.80 & 69.74 & 15.86 \\
\hline 5.5 & 2.351 & 15.32 & 3.77 & 4.08 & 75.29 & 16.51 \\
\hline 6.0 & 2.331 & 14.47 & 4.01 & 2.79 & 82.82 & 16.24 \\
\hline 6.5 & 2.282 & 13.38 & 4.06 & 2.44 & 85.39 & 16.70 \\
\hline
\end{tabular}
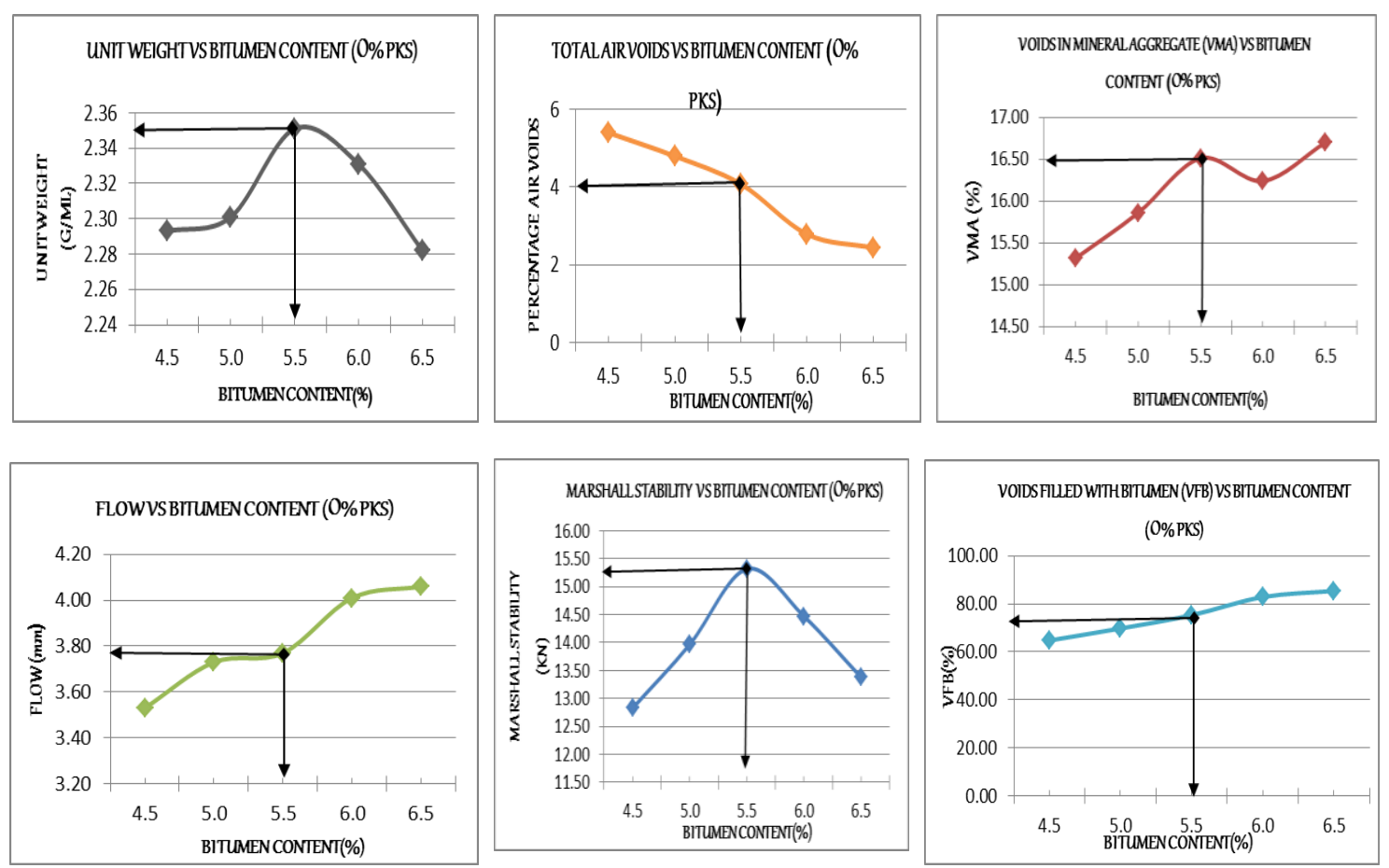

Fig. 2: Marshall Properties of Hot Mix Asphalt Prepared Using Zycotherm And 0\% PKS Partial Replacement as Coarse Aggregate Material (4-8mm) 
Table 4: The Optimum Binder Content Value At 0\% PKS Replacement

Average Value $=5.52 \%$

\begin{tabular}{|l|l|l|l|}
\hline & PROPERTIES & \multicolumn{2}{|l|}{$\begin{array}{l}\text { Median Of Percent Air } \\
\text { Voids(4\% Air Void) }\end{array}$} \\
\cline { 2 - 4 } & Max Unit Weight & Max. Stability & 5.55 \\
\hline BINDER CONTENT (\%) & 5.50 & 5.50 & 50 \\
\hline
\end{tabular}

Therefore, Optimum Binder Content (OBC) At 0\% PKS Replacement $=5.52 \%$

Table 5: Properties At 5.5\%Approx. Optimum Binder Content At 0\% PKS Replacement

\begin{tabular}{|l|l|l|l|}
\hline PROPERTIES & VALUES & SPECIFICATION & REMARKS \\
\hline Stability(KN) & 15.32 & Not Less Than 3.5 & Satisfactory \\
\hline Flow $(\mathrm{mm})$ & 3.77 & $2-6$ & Satisfactory \\
\hline Va $(\%)$ & 4.00 & $3-5$ & Satisfactory \\
\hline VFB $(\%)$ & 75.29 & $65-78$ & Satisfactory \\
\hline OBC $(\%)$ & 5.5 & $4.5-6.5$ & Satisfactory \\
\hline VMA (\%) & 16.51 & MINIMUM OF 13\% & Satisfactory \\
\hline
\end{tabular}

Table 6: Marshall Properties of Hot Mix Asphalt Prepared Using Zycotherm And 25\% PKS Replacement as Coarse Aggregate Material (4-8mm)

\begin{tabular}{|l|l|l|l|l|l|l|}
\hline $\begin{array}{l}\text { Binder } \\
\text { Content }(\%)\end{array}$ & $\begin{array}{l}\text { Unit Weight } \\
(\mathrm{g} / \mathrm{ml})\end{array}$ & $\begin{array}{l}\text { Marshall } \\
\text { Stability }(\mathrm{KN})\end{array}$ & $\begin{array}{l}\text { Flow } \\
(\mathrm{mm})\end{array}$ & $\begin{array}{l}\text { Total Air Voids } \\
(\mathrm{Va})(\%)\end{array}$ & $\begin{array}{l}\text { Voids Filled With } \\
\text { Bitumen }(\mathrm{VFB})(\%)\end{array}$ & $\begin{array}{l}\text { Voids In Mineral } \\
\text { Aggregate }(\mathrm{VMA})(\%)\end{array}$ \\
\hline 4.5 & 2.229 & 10.72 & 3.46 & 5.35 & 64.33 & 15.00 \\
\hline 5.0 & 2.279 & 11.17 & 3.58 & 5.04 & 68.50 & 16.00 \\
\hline 5.5 & 2.278 & 12.54 & 3.32 & 5.48 & 68.74 & 17.53 \\
\hline 6.0 & 2.317 & 12.62 & 3.78 & 2.98 & 81.77 & 16.35 \\
\hline 6.5 & 2.316 & 12.14 & 3.98 & 3.02 & 82.74 & 17.50 \\
\hline
\end{tabular}

Table 7: The Optimum Binder Content Value At 25\% PKS Replacement

\begin{tabular}{|l|l|l|l|}
\hline & \multicolumn{3}{|l|}{ PROPERTIES } \\
\cline { 2 - 4 } & Max Unit Weight & Max. Stability & $\begin{array}{l}\text { Median Of Percent Air } \\
\text { Voids(4\% Air Void) }\end{array}$ \\
\hline BINDER CONTENT $(\%)$ & 6.0 & 6.0 & 5.75 \\
\hline
\end{tabular}

Average Value $=5.92 \%$

Therefore, Optimum Binder Content (OBC) At 25\% PKS Replacement $=5.92 \%$

Table 8: Properties At 5.9\%Approx. Optimum Binder Content At 25\% PKS Replacement

\begin{tabular}{|l|l|l|l|}
\hline PROPERTIES & VALUES & SPECIFICATION & REMARKS \\
\hline Stability(KN) & 12.52 & Not Less Than 3.5 & Satisfactory \\
\hline Flow $(\mathrm{mm})$ & 3.79 & $2-6$ & Satisfactory \\
\hline Va $(\%)$ & 3.15 & $3-5$ & Satisfactory \\
\hline VFB $(\%)$ & 79.25 & $65-78$ & Not Satisfactory \\
\hline OBC $(\%)$ & 5.92 & $4.5-6.5$ & Satisfactory \\
\hline VMA $(\%)$ & 16.40 & MINIMUM OF 13\% & Satisfactory \\
\hline
\end{tabular}

Table 9: Marshall Properties of Hot Mix Asphalt Prepared Using Zycotherm And 50\% PKS Partial

Replacement as Coarse Aggregate Material (4-8mm)

\begin{tabular}{|l|l|l|l|l|l|l|}
\hline $\begin{array}{l}\text { Binder } \\
\text { Content }(\%)\end{array}$ & $\begin{array}{l}\text { Unit Weight } \\
(\mathrm{g} / \mathrm{ml})\end{array}$ & $\begin{array}{l}\text { Marshall } \\
\text { Stability }(\mathrm{KN})\end{array}$ & $\begin{array}{l}\text { Flow } \\
(\mathrm{mm})\end{array}$ & $\begin{array}{l}\text { Total Air Voids } \\
(\mathrm{Va})(\%)\end{array}$ & $\begin{array}{l}\text { Voids Filled With } \\
\text { Bitumen (VFB) }(\%)\end{array}$ & $\begin{array}{l}\text { Voids In Mineral } \\
\text { Aggregate }(\text { VMA) }(\%)\end{array}$ \\
\hline 4.5 & 2.202 & 10.81 & 3.67 & 6.18 & 61.59 & 16.09 \\
\hline 5.0 & 2.202 & 10.98 & 3.74 & 7.56 & 58.35 & 18.15 \\
\hline 5.5 & 2.218 & 11.59 & 4.00 & 3.44 & 77.32 & 15.17 \\
\hline 6.0 & 2.222 & 11.94 & 3.99 & 3.10 & 80.53 & 15.92 \\
\hline 6.5 & 2.249 & 11.56 & 4.01 & 2.34 & 85.73 & 16.40 \\
\hline
\end{tabular}

Table 10: The Optimum Binder Content Value At 50\% PKS Replacement

Average Value $=5.99 \%$

\begin{tabular}{|l|l|l|l|}
\hline \multirow{2}{*}{} & PROPERTIES & \multicolumn{2}{l|}{$\begin{array}{l}\text { Median Of Percent Air } \\
\text { Voids(4\% Air Void) }\end{array}$} \\
\cline { 2 - 4 } & Max Unit Weight & Max. Stability & 5.48 \\
\hline BINDER CONTENT (\%) & 6.5 & 6.0 & 5.48 \\
\hline
\end{tabular}

Therefore, Optimum Binder Content (OBC) At 50\% PKS Replacement $=5.99 \%$

Table 11: Properties At 6.0\%Approx. Optimum Binder Content At 50\% PKS Replacement

\begin{tabular}{|l|l|l|l|}
\hline PROPERTIES & VALUES & SPECIFICATION & REMARKS \\
\hline Stability $(\mathrm{KN})$ & 11.94 & Not Less Than 3.5 & Satisfactory \\
\hline Flow $(\mathrm{mm})$ & 3.99 & $2-6$ & Satisfactory \\
\hline
\end{tabular}


*Study of the performance of graded palm kernel shells $(P K S)$ as partial replacement for coarse......*

\begin{tabular}{|l|l|l|l|}
\hline Va (\%) & 3.10 & $3-5$ & Satisfactory \\
\hline VFB $(\%)$ & 80.03 & $65-78$ & Not Satisfactory \\
\hline OBC $(\%)$ & 5.99 & $4.5-6.5$ & Satisfactory \\
\hline VMA $(\%)$ & 15.92 & MINIMUM OF 13\% & Satisfactory \\
\hline
\end{tabular}

Table 12: Marshall Properties of Hot Mix Asphalt Prepared Using Zycotherm And 75\% PKS Partial Replacement as Coarse Aggregate Material (4-8mm)

\begin{tabular}{|l|l|l|l|l|l|l|}
\hline $\begin{array}{l}\text { Binder } \\
\text { Content }(\%)\end{array}$ & $\begin{array}{l}\text { Unit Weight } \\
(\mathrm{g} / \mathrm{ml})\end{array}$ & $\begin{array}{l}\text { Marshall } \\
\text { Stability }(\mathrm{KN})\end{array}$ & $\begin{array}{l}\text { Flow } \\
(\mathrm{mm})\end{array}$ & $\begin{array}{l}\text { Total Air Voids } \\
(\mathrm{Va})(\%)\end{array}$ & $\begin{array}{l}\text { Voids Filled With } \\
\text { Bitumen (VFB) }(\%)\end{array}$ & $\begin{array}{l}\text { Voids In Mineral } \\
\text { Aggregate }(\mathrm{VMA})(\%)\end{array}$ \\
\hline 4.5 & 2.085 & 10.39 & 3.38 & 3.96 & 69.49 & 12.98 \\
\hline 5.0 & 2.103 & 9.92 & 3.62 & 4.41 & 69.63 & 14.52 \\
\hline 5.5 & 2.123 & 10.40 & 3.83 & 5.05 & 68.98 & 16.28 \\
\hline 6.0 & 2.143 & 11.07 & 3.88 & 2.15 & 85.18 & 14.51 \\
\hline 6.5 & 2.109 & 10.49 & 3.78 & 3.70 & 78.08 & 16.88 \\
\hline
\end{tabular}

Table 13: The Optimum Binder Content Value At 75\% PKS Replacement

\begin{tabular}{|l|l|l|l|}
\hline & PROPERTIES & \multicolumn{2}{|c|}{} \\
\cline { 2 - 4 } & Max Unit Weight & Max. Stability & $\begin{array}{l}\text { Median Of Percent Air } \\
\text { Voids(4\% Air Void) }\end{array}$ \\
\hline BINDER CONTENT (\%) & 6.5 & 6.0 & 4.55 \\
\hline
\end{tabular}

Average Value $=5.52 \%$

Therefore, Optimum Binder Content (OBC) At 75\% PKS Replacement $=5.52 \%$

Table 14: Properties At 5.5\%Approx. Optimum Binder Content At 75\% PKS Replacement

\begin{tabular}{|l|l|l|l|}
\hline PROPERTIES & VALUES & SPECIFICATION & REMARKS \\
\hline Stability(KN) & 10.40 & Not Less Than 3.5 & Satisfactory \\
\hline Flow $(\mathrm{mm})$ & 3.83 & $2-6$ & Satisfactory \\
\hline Va $(\%)$ & 4.99 & $3-5$ & Satisfactory \\
\hline VFB $(\%)$ & 68.98 & $65-78$ & Satisfactory \\
\hline OBC $(\%)$ & 5.52 & $4.5-6.5$ & Satisfactory \\
\hline VMA (\%) & 15.00 & MINIMUM OF 13\% & Satisfactory \\
\hline
\end{tabular}

Table 15: Marshall Properties of hot mix asphalt prepared using zycotherm and 100\% PKS Partial Replacement

\begin{tabular}{|c|c|c|c|c|c|c|}
\hline $\begin{array}{l}\text { Binder } \\
\text { Content }(\%)\end{array}$ & $\begin{array}{l}\text { Unit Weight } \\
(\mathrm{g} / \mathrm{ml})\end{array}$ & $\begin{array}{l}\text { Marshall } \\
\text { Stability }(\mathrm{KN})\end{array}$ & $\begin{array}{l}\text { Flow } \\
(\mathrm{mm})\end{array}$ & $\begin{array}{l}\text { Total Air Voids } \\
(\text { Va) }(\%)\end{array}$ & $\begin{array}{l}\text { Voids Filled With } \\
\text { Bitumen (VFB) (\%) }\end{array}$ & $\begin{array}{l}\text { Voids In Mineral } \\
\text { Aggregate (VMA) (\%) }\end{array}$ \\
\hline 4.5 & 2.126 & 9.84 & 3.78 & 5.97 & 60.65 & 15.17 \\
\hline 5.0 & 2.114 & 10.03 & 3.74 & 6.95 & 59.38 & 17.11 \\
\hline 5.5 & 2.101 & 10.09 & 3.71 & 4.15 & 72.81 & 15.26 \\
\hline 6.0 & 2.080 & 9.75 & 3.60 & 4.46 & 72.90 & 16.46 \\
\hline 6.5 & 2.118 & 9.82 & 3.59 & 2.53 & 83.96 & 15.77 \\
\hline
\end{tabular}

Table 16: The Optimum Binder Content Value At 100\% PKS Replacement

Average Value $=5.27 \%$

\begin{tabular}{|l|l|l|l|}
\hline & PROPERTIES & $\begin{array}{l}\text { Median Of Percent Air } \\
\text { Voids(4\% Air Void) }\end{array}$ \\
\cline { 2 - 4 } & Max Unit Weight & Max. Stability & 5.5 \\
\hline BINDER CONTENT $(\%)$ & 4.5 & 5.5 & \\
\hline
\end{tabular}

Therefore, Optimum Binder Content (OBC) At 100\% PKS Replacement $=5.27 \%$

Table 17: Properties At 5.3\%Approx. Optimum Binder Content At 100\% PKS Replacement

\begin{tabular}{|l|l|l|l|}
\hline PROPERTIES & VALUES & SPECIFICATION & REMARKS \\
\hline Stability(KN) & 10.07 & Not Less Than 3.5 & Satisfactory \\
\hline Flow $(\mathrm{mm})$ & 3.70 & $2-6$ & Satisfactory \\
\hline Va $(\%)$ & 5.00 & $3-5$ & Satisfactory \\
\hline VFB $(\%)$ & 70.00 & $65-78$ & Satisfactory \\
\hline OBC $(\%)$ & 5.27 & $4.5-6.5$ & Satisfactory \\
\hline VMA $(\%)$ & 16.00 & MINIMUM OF 13\% & Satisfactory \\
\hline
\end{tabular}

Table 18: Comparisons Of Properties At Mix Proportions Of PKS Replacement

\begin{tabular}{|l|l|l|l|l|l|l|}
\hline PROPERTIES & $\mathbf{0 \%}$ & $\mathbf{2 5 \%}$ & $\mathbf{5 0 \%}$ & $\mathbf{7 5 \%}$ & $\mathbf{1 0 0 \%}$ & SPECIFICATION \\
\hline Stability $(\mathrm{KN})$ & 15.32 & 12.52 & 11.94 & 10.40 & 10.07 & Not Less Than 3.5 \\
\hline Flow(mm) & 3.77 & 3.79 & 3.99 & 3.83 & 3.70 & $2-6$ \\
\hline Va $(\%)$ & 4.00 & 3.15 & 3.10 & 4.99 & 5.00 & $3-5$ \\
\hline VFB $(\%)$ & 75.29 & 79.25 & 80.03 & 68.98 & 70.00 & $65-78$ \\
\hline OBC (\%) & 5.5 & 5.92 & 5.99 & 5.52 & 5.27 & $4.5-6.5$ \\
\hline VMA $(\%)$ & 16.51 & 16.40 & 15.92 & 15.00 & 16.00 & MINIMUM OF 13\% \\
\hline
\end{tabular}


Table 19: Marshall Stability Parameter Variation From 0\% - 100\% PKS Replacement

\begin{tabular}{|c|c|c|c|c|c|c|c|c|}
\hline BINDER & $0 \%$ & $25 \%$ & $50 \%$ & $75 \%$ & $100 \%$ & \multicolumn{3}{|c|}{ SPECIFICATION } \\
\hline 4.5 & 12.83 & 10.72 & 10.81 & 10.39 & 9.84 & \multirow{5}{*}{$\begin{array}{l}\text { LIGHT } \\
\text { TRAFFIC } \\
\left(<10^{4} \text { ESALS }\right) \\
\text { MIN. } \\
3.5 \mathrm{KN}\end{array}$} & \multirow{5}{*}{$\begin{array}{l}\text { MEDIUM } \\
\text { TRAFFIC } \\
\left(10^{4}-10^{6}\right. \\
\text { ESALS }) \\
\text { MIN 60KN }\end{array}$} & \multirow{5}{*}{$\begin{array}{l}\text { HEAVY } \\
\text { TRAFFIC } \\
\left(>\quad 10^{6}\right. \\
\text { ESALS) } \\
\text { MIN 9 0KN }\end{array}$} \\
\hline 5.0 & 13.97 & 11.17 & 10.98 & 9.92 & 10.03 & & & \\
\hline 5.5 & 15.32 & 12.54 & 11.59 & 10.40 & 10.09 & & & \\
\hline 6.0 & 14.47 & 12.62 & 11.94 & 11.07 & 9.75 & & & \\
\hline 6.5 & 13.38 & 12.14 & 11.56 & 10.49 & 9.82 & & & \\
\hline
\end{tabular}

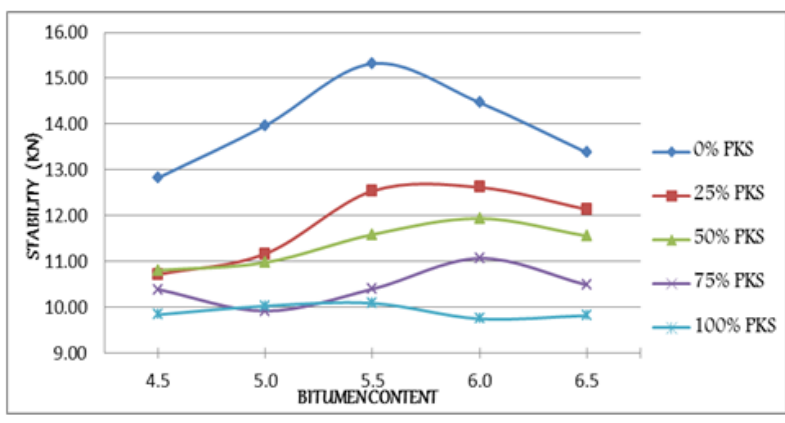

It is observed that there is decrease in stability from $0 \%$ to $100 \% \mathrm{mix}$ proportions of PKS replacement. Stability has peak value of $15.32 \mathrm{KN}$ at $5.5 \mathrm{BC} \%$ of $0 \% \mathrm{PKS}$ replacement. Stability has lowest value of $9.82 \mathrm{KN}$ at $6.5 \mathrm{BC} \%$ of $100 \%$ PKS replacement. The trend shows about $35 \%$ reduction in stability but the lowest value obtained is still within the specified limit

Fig. 3: Graph of Marshall Stability Variations From 0\% To 100\% PKS Replacement

Table 20: Marshall Flow Parameter Variations From 0\% - 100\% PKS Replacement

\begin{tabular}{|c|c|c|c|c|c|c|}
\hline BINDER CONTENT (\%) & $0 \%$ & $25 \%$ & $50 \%$ & $75 \%$ & $100 \%$ & SPECIFICATION \\
\hline 4.5 & 3.53 & 3.46 & 3.67 & 3.38 & 3.78 & \multirow[t]{5}{*}{$(2 \mathrm{Mm}-4 \mathrm{Mm})$} \\
\hline 5.0 & 3.73 & 3.58 & 3.74 & 3.62 & 3.74 & \\
\hline 5.5 & 3.77 & 3.32 & 4.00 & 3.83 & 3.71 & \\
\hline 6.0 & 4.10 & 3.78 & 3.99 & 3.88 & 3.60 & \\
\hline 6.5 & 4.06 & 3.98 & 4.02 & 3.78 & 3.59 & \\
\hline
\end{tabular}

(Note: Results Obtained Not Within Limits Are In Red)

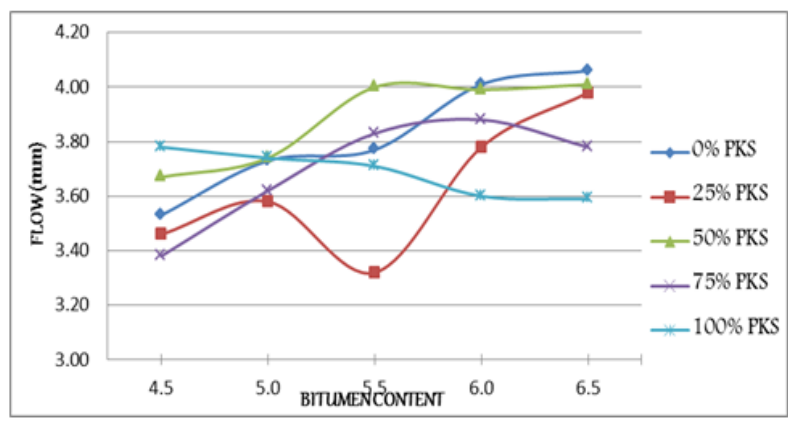

The highest Marshall flow was $4.06 \mathrm{~mm}$ at $6.5 \mathrm{BC}$ of $0 \% \mathrm{PKS}$ and the least was $3.32 \mathrm{~mm}$ at $5.5 \mathrm{BC}$ of $25 \%$ PKS. All flow parameters were within specified limits for all varying rates of PKS replacement except for marginal increases in $50 \% \mathrm{PKS}$ and $0 \% \mathrm{PKS}$.

Fig. 4: Graph of Marshall Flow Variations From 0\% To 100\% PKS Replacement

Table 21: Unit Weight Parameter Variations From 0\% - 100\% Pks Replacement

\begin{tabular}{|l|l|l|l|l|l|l|}
\hline BINDER CONTENT $(\boldsymbol{\%})$ & $\mathbf{0 \%}$ & $\mathbf{2 5 \%}$ & $\mathbf{5 0 \%}$ & $\mathbf{7 5 \%}$ & $\mathbf{1 0 0 \%}$ & SPECIFICATION \\
\hline 4.5 & 2.293 & 2.229 & 2.202 & 2.085 & 2.126 & \multirow{2}{*}{ N/A } \\
\cline { 1 - 6 } 5.0 & 2.301 & 2.279 & 2.202 & 2.103 & 2.114 & \\
\cline { 1 - 5 } 6.5 & 2.351 & 2.278 & 2.218 & 2.123 & 2.101 & \\
\cline { 1 - 5 } 6.5 & 2.331 & 2.317 & 2.222 & 2.143 & 2.080 & \\
\hline
\end{tabular}

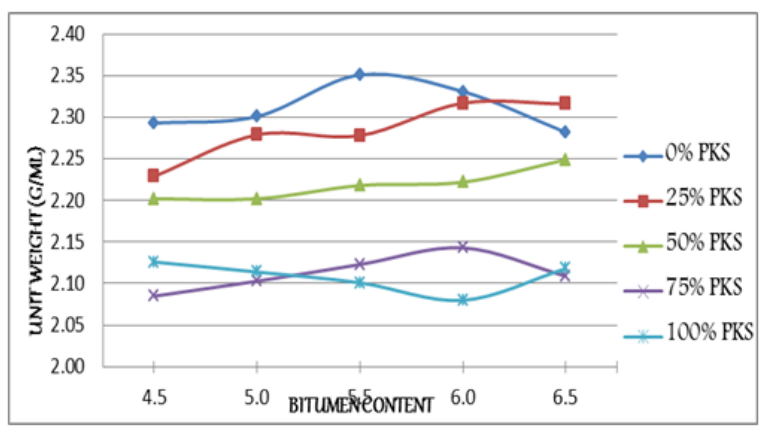

It is observed as expected that there was decrease in the unit weight from $0 \%$ to $100 \% \mathrm{mix}$ proportions of PKS

replacement. This is as a result of the lower specific density of Palm kemel shells.

Fig. 5: Graph of Unit Weight Variations From 0\% To 100\% PKS Replacement 
Table 22: Percentage Air Void (Va) Parameter Variation From 0\% - 100\% PKS Replacement

\begin{tabular}{|l|l|l|l|l|l|l|}
\hline BINDER CONTENT (\%) & $\mathbf{0 \%}$ & $\mathbf{2 5 \%}$ & $\mathbf{5 0 \%}$ & $\mathbf{7 5 \%}$ & $\mathbf{1 0 0 \%}$ & \multirow{2}{*}{ SPECIFICATION } \\
\hline 4.5 & 5.40 & 5.35 & 6.18 & 3.96 & 5.97 & \multirow{3}{*}{$\mathbf{3}-\mathbf{5} \%$} \\
\cline { 1 - 6 } 5.0 & 4.80 & 5.04 & 7.56 & 4.41 & 6.95 & \\
\cline { 1 - 6 } & 4.08 & 5.48 & 3.44 & 5.05 & 4.15 & \\
\cline { 1 - 5 } 6.0 & 2.79 & 2.98 & 3.10 & 2.15 & 4.46 & \\
\cline { 1 - 5 } & 2.44 & 3.02 & 2.34 & 3.70 & 2.53 & \\
\hline
\end{tabular}

(Note: Results Obtained Not Within Limits Are In Red)

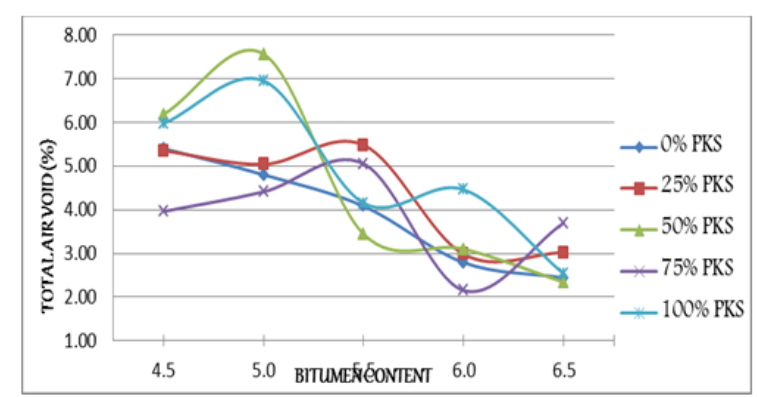

The air voids seemed to reduce with increase in binder content across all varying rates. Highest value is at $5.0 \% \mathrm{BC}$ of $50 \%$ PKS whereas the least value was at $6.0 \% \mathrm{BC}$ of $75 \%$ PKS

Fig. 6: Graph of Air Void (\%) Variations From 0\% To 100\% PKS Replacement

TABLE 23: Voids In Mineral Aggregates (VMA) Variations From 0\% - 100\% PKS Replacement

\begin{tabular}{|c|c|c|c|c|c|c|}
\hline BINDER CONTENT (\%) & 0\% & $25 \%$ & $50 \%$ & $75 \%$ & $100 \%$ & SPECIFICATION \\
\hline 4.5 & 15.32 & 15.00 & 16.09 & 12.98 & 15.17 & \multirow[t]{5}{*}{ MINIMUM OF $13 \%$} \\
\hline 5.0 & 15.86 & 16.00 & 18.15 & 14.52 & 17.11 & \\
\hline 5.5 & 16.51 & 17.53 & 15.17 & 16.28 & 15.26 & \\
\hline 6.0 & 16.24 & 16.35 & 15.92 & 14.51 & 16.46 & \\
\hline 6.5 & 16.70 & 17.50 & 16.40 & 16.88 & 15.77 & \\
\hline
\end{tabular}

(Note: Results Obtained Not Within Limits Are In Red)

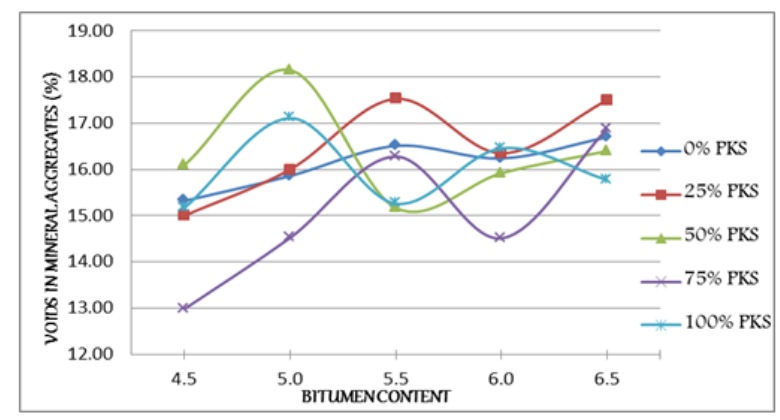

Highest VMA result of $18.15 \%$ was at $5.0 \% \mathbf{B C}$ at $50 \%$ PKS replacement, whereas the minimum result of $14.52 \%$ is at $6.0 \%$ BC at $75 \%$ PKS replacement.

Fig. 7: Voids in Mineral Aggregates (\%) Variations From 0\% To 100\% PKS Replacement

Table 24: Voids Filled With Bitumen (VFB) Variation From 0\% - 100\% PKS Replacement

\begin{tabular}{|c|c|c|c|c|c|c|c|c|}
\hline BINDER CONTENT $(\%)$ & 0\% & $25 \%$ & $50 \%$ & $75 \%$ & $100 \%$ & \multicolumn{3}{|c|}{ SPECIFICATION } \\
\hline 4.5 & 64.75 & 64.33 & 61.59 & 69.49 & 60.65 & \multirow{5}{*}{$\begin{array}{l}\text { LIGHT } \\
\text { TRAFFIC } \\
\left(<\quad 10^{4}\right. \\
\text { ESALS) } \\
70-80\end{array}$} & \multirow{5}{*}{$\begin{array}{l}\text { MEDIUM } \\
\text { TRAFFIC } \\
\left(10^{4}-10^{6}\right. \\
\text { ESALS }) \\
65-78\end{array}$} & \multirow{5}{*}{$\begin{array}{l}\text { HEAVY } \\
\text { TRAFFIC } \\
\left(>\quad 10^{6}\right. \\
\text { ESALS) } \\
65-75\end{array}$} \\
\hline 5.0 & 69.74 & 68.50 & 58.35 & 69.63 & 59.38 & & & \\
\hline 5.5 & 75.29 & 68.74 & 77.32 & 68.98 & 72.81 & & & \\
\hline 6.0 & 82.82 & 81.77 & 80.53 & 85.18 & 72.90 & & & \\
\hline 6.5 & 85.39 & 82.74 & 85.73 & 78.08 & 83.96 & & & \\
\hline
\end{tabular}
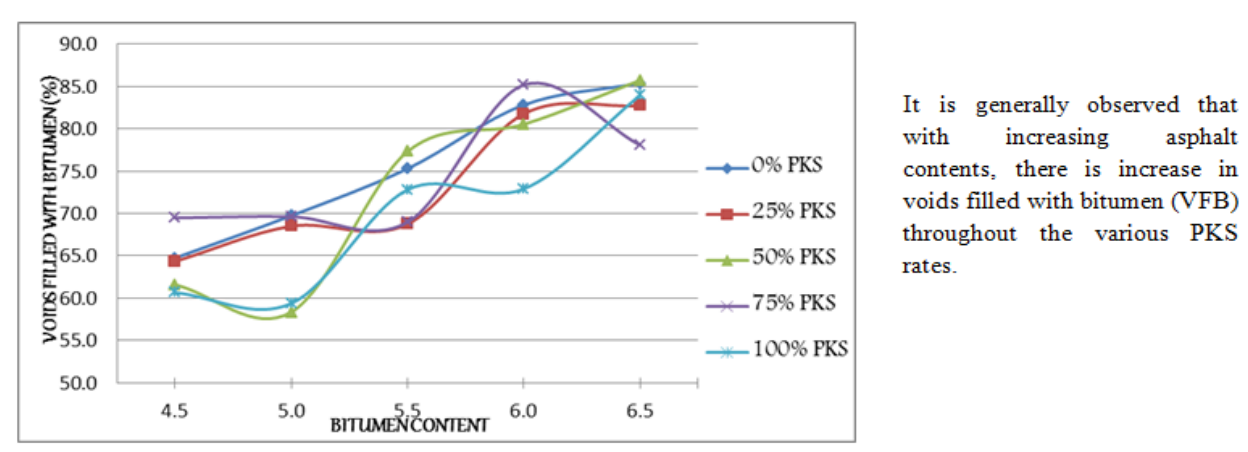

Fig. 8: Voids Filled With Bitumen (\%) Variations From 0\% To 100\% PKS Replacement 
Table 25: Comparison of Some Asphalt Properties of the Zycotherm Modified Bitumen and Plain Bitumen.

\begin{tabular}{|l|l|l|l|}
\hline ASPHALT PROPERTY & $\begin{array}{l}\text { PLAIN } \\
\text { BITUMEN }\end{array}$ & $\begin{array}{l}\text { ZYCOTHERM } \\
\text { MODIFIED BITUMEN }\end{array}$ & SPECIFICATIONS \\
\hline Specific Gravity At $25^{\circ} \mathrm{c}$ & 1.03 & 1.04 & $\geq 1$ \\
\hline Softening Point (Ball \& Ring) ${ }^{\circ} \mathrm{C}$ & 52 & 51 & $>50^{\circ} \mathrm{c}$ \\
\hline Penetration At $25 \mathrm{C}-0.1 \mathrm{~mm}$ & $60 / 70$ & $60 / 70$ & $60-70$ \\
\hline Solubility In Trichloroethylene \% By Weight & 99 & 99 & 99 \\
\hline
\end{tabular}

Table 26: Comparisons of Asphalt Concrete Marshall Properties of Zycotherm Modified Bitumen and the Plain Bitumen (0\% PKS Replacement) At OBC.

\begin{tabular}{|c|c|c|c|}
\hline PROPERTIES & $\begin{array}{l}\text { PLAIN BITUMEN } \\
(0 \% \text { PKS })\end{array}$ & $\begin{array}{l}\text { ZYCOTHERM MODIFIED } \\
\text { BITUMEN (0\% PKS) }\end{array}$ & SPECIFICATION \\
\hline STABILITY(KN) & 14.59 & 15.32 & MINIMUM OF 3.5 \\
\hline FLOW(Mm) & 3.64 & 3.77 & $2-4$ \\
\hline VA (\%) & 5.70 & 4.00 & $3-5$ \\
\hline VFB (\%) & 69.9 & 75.29 & $65-78$ \\
\hline $\mathrm{OBC}(\%)$ & 5.70 & 5.52 & $4.5-6.5$ \\
\hline VMA (\%) & 18.70 & 16.51 & MINIMUM OF $13 \%$ \\
\hline
\end{tabular}

Table 27: Comparison of Asphalt Concrete Marshall Stability of Zycotherm Modified Bitumen and the Plain Bitumen At 0\% PKS Replacement

\begin{tabular}{|c|c|c|c|c|c|}
\hline & \multicolumn{2}{|c|}{ MARSHALL STABILITY OBTAINED IN (KN) } & & & \\
\hline $\begin{array}{l}\text { BINDER } \\
\text { CONTENT }(\%)\end{array}$ & $\begin{array}{l}\text { PLAIN BITUMEN } \\
(0 \% \text { PKS })\end{array}$ & $\begin{array}{l}\text { ZYCOTHERM MODIFIED } \\
\text { BITUMEN (0\% PKS) }\end{array}$ & \multicolumn{3}{|c|}{ SPECIFICATION } \\
\hline 4.5 & 13.73 & 12.83 & \multirow{5}{*}{ 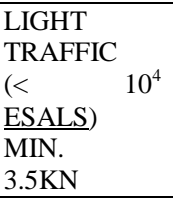 } & \multirow{5}{*}{$\begin{array}{l}\text { MEDIUM } \\
\text { TRAFFIC } \\
\left(10^{4}-10^{6}\right. \\
\text { ESALS }) \\
\text { MIN. } \\
6.0 \mathrm{KN}\end{array}$} & \multirow{5}{*}{$\begin{array}{l}\text { HEAVY } \\
\text { TRAFFIC } \\
\left(>\quad 10^{6}\right. \\
\text { ESALS) } \\
\text { MIN. } \\
9.0 \mathrm{KN}\end{array}$} \\
\hline 5.0 & 15.05 & 13.97 & & & \\
\hline 5.5 & 15.78 & 15.32 & & & \\
\hline 6.0 & 12.81 & 14.47 & & & \\
\hline 6.5 & 12.38 & 13.38 & & & \\
\hline
\end{tabular}

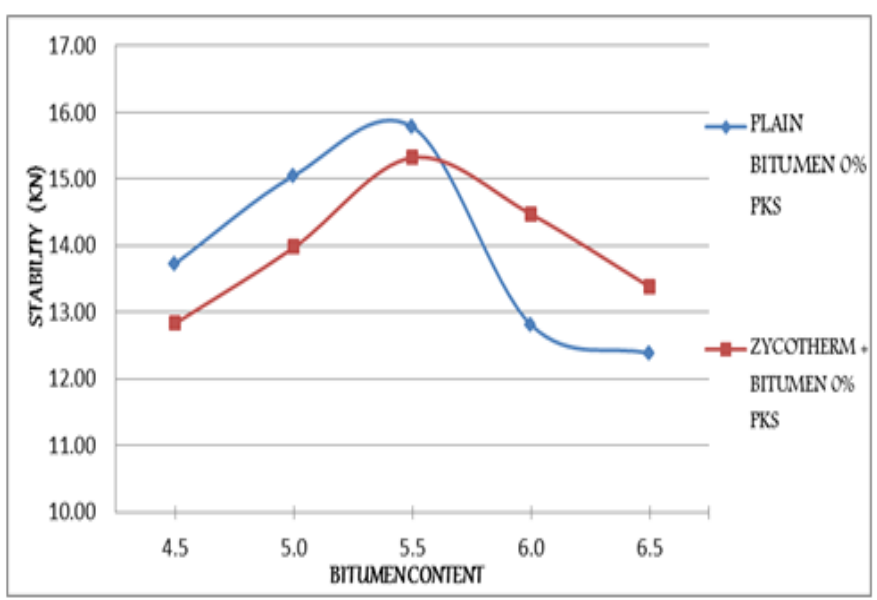

It was however observed that between $4.5 \%-5.5 \% \mathrm{BC}$, Marshall Stability was slightly higher with the plain bitumen. For 6.0 and $6.5 \% \mathrm{BC}$ the stability values were much higher with the zycotherm modified bitumen. All stability results obtained were well over the required standard limits.

Fig. 9: Graph of Stability (KN) Variations between the Plain and Zycotherm Modified Bitumen

Table 28: Comparison of Asphalt Concrete Marshall Flow of Zycotherm Modified Bitumen and Plain Bitumen At $0 \%$ PKS Replacement

\begin{tabular}{|c|c|c|c|}
\hline & \multicolumn{2}{|c|}{ MARSHALL FLOW STABILITY OBTAINED IN (Mm) } & \\
\hline $\begin{array}{l}\text { BINDER CONTENT } \\
(\%)\end{array}$ & $\begin{array}{l}\text { PLAIN BITUMEN } \\
(0 \% \text { PKS })\end{array}$ & $\begin{array}{ll}\text { ZYCOTHERM } & \text { MODIFIED } \\
\text { BITUMEN (0\%PKS) } & \\
\end{array}$ & SPECIFICATION \\
\hline 4.5 & 3.23 & 3.53 & $2-4(\mathrm{Mm})$ \\
\hline 5.0 & 3.32 & 3.73 & \\
\hline 5.5 & 3.60 & 3.77 & \\
\hline 6.0 & 3.75 & 4.01 & \\
\hline 6.5 & 3.92 & 4.06 & \\
\hline
\end{tabular}

(Note: Results Obtained Not Within Limits Are In Red) 


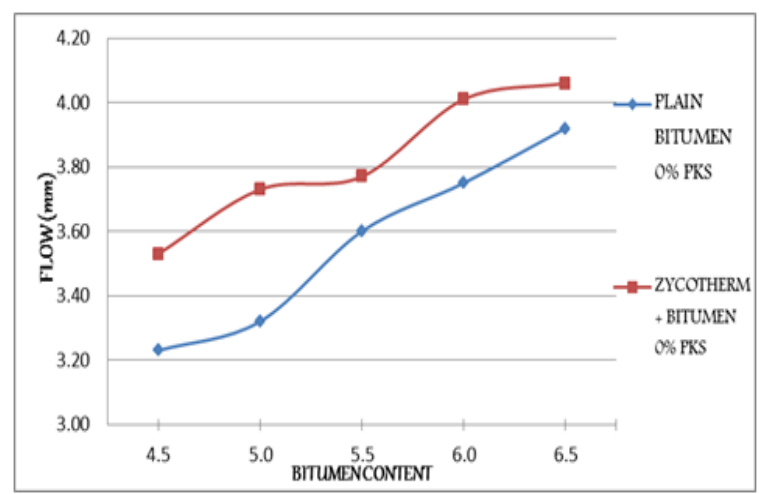

The result showed higher flow rates from the zycotherm modified bitumen more than that of the Plain bitumen across all the various binder content percentages.

Fig. 10: Graph of Flow (mm) Variations between the Plain and Zycotherm Modified Bitumen

TABLE-29: Comparison of Asphalt Concrete Marshall Air Voids of Zycotherm Modified Bitumen and Plain Bitumen At 0\% PKS Replacement

\begin{tabular}{|c|c|c|c|}
\hline & \multicolumn{2}{|c|}{ TOTAL AIR VOIDS(VA) PERCENT OBTAINED } & \\
\hline $\begin{array}{l}\text { BINDER } \\
\text { CONTENT }(\%)\end{array}$ & PLAIN BITUMEN (0\% PKS) & $\begin{array}{l}\text { ZYCOTHERM MODIFIED } \\
\text { BITUMEN (0\% PKS) }\end{array}$ & SPECIFICATION \\
\hline 4.5 & 6.1 & 5.40 & $3-5(\%)$ \\
\hline 5.0 & 5.9 & 4.80 & \\
\hline 5.5 & 5.7 & 4.08 & \\
\hline 6.0 & 5.6 & 2.79 & \\
\hline 6.5 & 4.8 & 2.44 & \\
\hline
\end{tabular}

(Note: Results Obtained Not Within Limits Are In Red)



The results obtained shows reductions in percent air void in the zycotherm modified bitumen with regards to the plain bitumen results.

Fig. 11: Graph of Air Void (\%) Variations between the Plain and Zycotherm Modified Bitumen

TABLE- 30: Comparison of Asphalt Concrete Marshall Unit Weight (g/ml) Of Zycotherm Modified Bitumen and Plain Bitumen At 0\% PKS Replacement

\begin{tabular}{|c|c|c|c|}
\hline & \multicolumn{2}{|c|}{ UNIT WEIGHT (G/ML) OBTAINED } & \\
\hline BINDER CONTENT (\%) & $\begin{array}{l}\text { PLAIN BITUMEN } \\
(0 \% \text { PKS })\end{array}$ & $\begin{array}{l}\text { ZYCOTHERM MODIFIED } \\
\text { BITUMEN (0\%PKS) }\end{array}$ & SPECIFICATION \\
\hline 4.5 & 2.392 & 2.293 & N/A \\
\hline 5.0 & 2.397 & 2.301 & \\
\hline 5.5 & 2.419 & 2.351 & \\
\hline 6.0 & 2.389 & 2.331 & \\
\hline 6.5 & 2.372 & 2.282 & \\
\hline
\end{tabular}

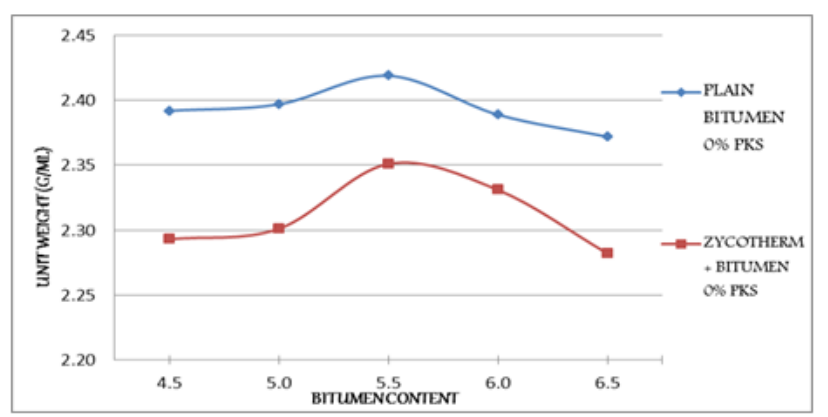

The results show a decrease in the unit weight, with the unit weight obtained using the plain bitumen were relatively higher than the values obtained from the modified bitumen. This is because the zycotherm additive forms a coating on the aggregates reducing water and air absorption rates hence reducing unit weights of compacted samples.

Fig. 12: Graph of Unit Weight $(\mathrm{g} / \mathrm{ml})$ Variations between the Plain and Zycotherm Modified Bitumen 
TABLE-31: Comparison of Asphalt Concrete Marshall Voids in Mineral Aggregate Vma (\%) Of Zycotherm Modified Bitumen and Plain Bitumen At 0\% PKS Replacement

\begin{tabular}{|c|c|c|c|}
\hline & \multicolumn{2}{|c|}{ VOIDS IN MINERAL AGGREGATES (\%) OBTAINED } & \multirow[b]{2}{*}{ SPECIFICATION } \\
\hline $\begin{array}{l}\text { BINDER } \\
\text { CONTENT (\%) }\end{array}$ & PLAIN BITUMEN (0\% PKS) & $\begin{array}{l}\text { ZYCOTHERM MODIFIED } \\
\text { BITUMEN (0\% PKS) }\end{array}$ & \\
\hline 4.5 & 16.6 & 15.32 & \multirow[t]{5}{*}{ MINIMUM OF $13 \%$} \\
\hline 5.0 & 17.5 & 15.86 & \\
\hline 5.5 & 18.6 & 16.51 & \\
\hline 6.0 & 19.5 & 16.24 & \\
\hline 6.5 & 19.8 & 16.70 & \\
\hline
\end{tabular}

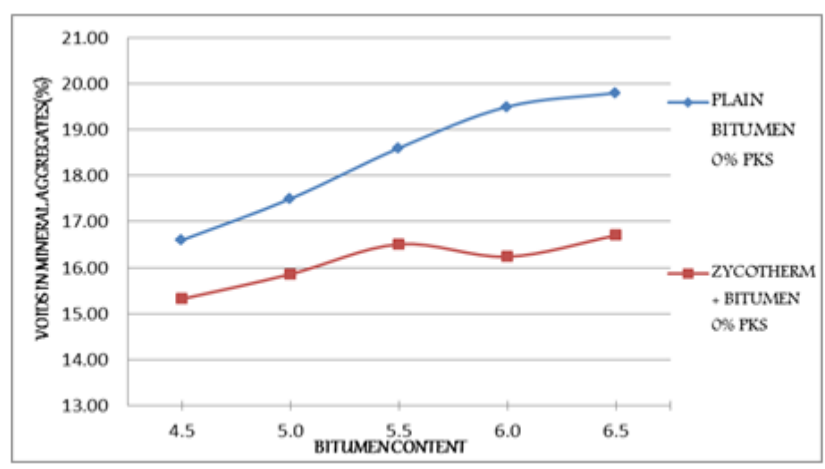

The results show a trend of increase in value of VMA as the binder contents are increased; however the results of VMA show reduction in values as regards the modified bitumen.

Fig. 13: Graph of VMA (\%) Variations between the Plain and Zycotherm Modified Bitumen

TABLE-32: Comparison of Asphalt Concrete Marshall Voids Filled With Bitumen (VFB) of Zycotherm Modified Bitumen and Plain Bitumen At 0\% PKS Replacement

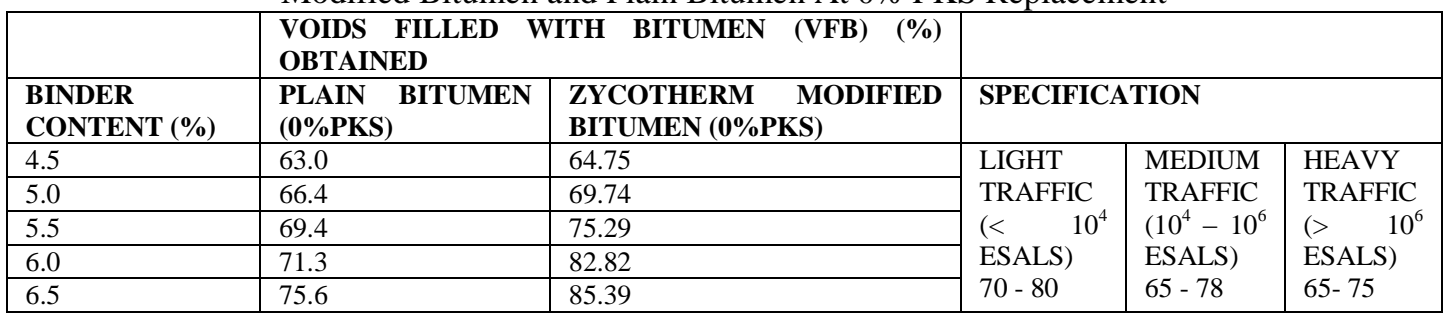

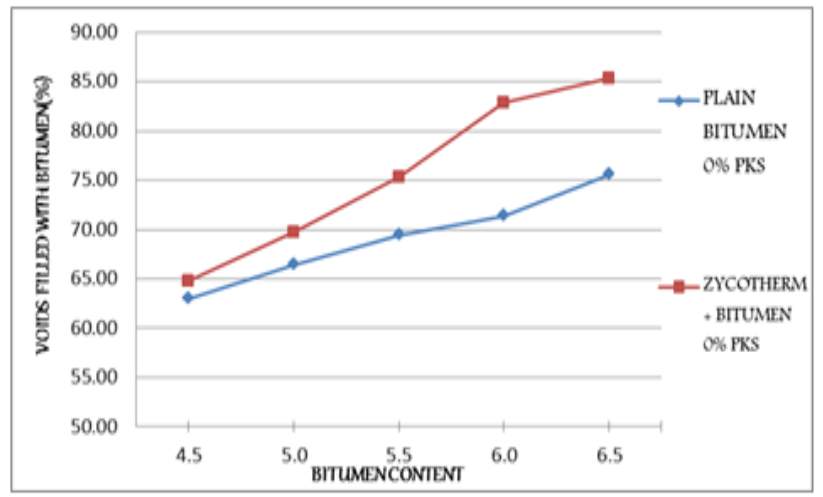

VFB result variations obtained using both sets of bitumen as volume of voids filled with bitumen in the modified asphalt concrete was higher than that of the plain bitumen.

Fig. 14: Graph of VFB (\%) Variation between the Plain and Zycotherm Modified Bitumen

\section{CONCLUSIONS}

From the research work results, it shows that PKS can be a viable source of coarse aggregate for partial replacement in asphalt concrete. Partial replacements at $75 \%$ and $100 \%$ of the $4-8 \mathrm{~mm}$ coarse aggregate portion at optimum binder contents seem to be the best options as both mix result values meet the specifications as regards the conditions for use for light - heavily trafficked roads. However, we cannot rule out the effects of the modifier as the modification of the binder with zycotherm chemical additive seemed to have helped to this effect. This is as test results show that the zycotherm additive also improves asphalt concrete mixtures especially the void characteristics of the mix and is recommended to use in practice. 


\section{REFERENCES}

[1]. Adewale Olutaiwo And Adeniyi Owolabi (2015); "Investigation Of The Effects Of Partial Replacement Of Coarse Aggregates With Graded Palm Kernel Shells In Asphaltic Binder Course”; British Journal Of Applied Science And Technology; 10(3):1-13,2015 Article No BJAST.18785;ISSN:2251-0843,Science Domain International; Www.Sciencedomain.Org

[2]. A. Shik Bellary And Lokesh Gupta; Laboratory Studies On Warm Bituminous Concrete Mix Prepared Using GGBS As Filler Material And Zycotherm As Additive; International Journal Of Advanced Technology In Engineering And Science Vol. No. 4, Special Issue No. 01 May 2016, Www.Ijates.Com, 1464436765-1841jates.Pdf

[3]. Bamidele I.O Dahunsi, Olufemi.S. Awogboro, Mutiu Akinpolu And Oladipupo S.O Olafusi (2013); Investigation Of The Properties Of Pure Water Sachet Modified Bitumen, Published By The International Institute For Science, Technology And Education (IISTE); Civil And Environmental Research Www.Iiste.Org ISSN 2222-1719 (Paper) ISSN 2222-2863 (Online) Vol.3, No.2, 2013

[4]. G.R. Harish And M.N. Shirakumar (2013); Performance Evaluation Of Bituminous Concrete Incorporating Crumb Rubber And Waste Shredded Thermoplastics; IJRET: International Journal Of Research In Engineering Technology; ISSN: 2319.1163/Pissn: 2321-7308

[5]. Mangesh. V. Madurwar, Sachin Arvind Mandavgane and Rahul. V. Ralegaonkar (2012); Application Of Agro-Waste For Suitable Construction Materials; Review Article In Construction And Building Materials; October 2012

[6]. Mohammed. H, Afolabi. K O And Umoru. L. E(2014); Crushed Palm Kernel Shell As Partial Replacement Of Fine Aggregate In Asphaltic Concrete; International Journal Of Materials, Methods And Technologies, Volume 2, No 1,January 2014, Pp. 1- 5; ISSN: 2327-0322.

[7]. Peter. N. Ndoke (2006); Performance Of Palm Kernel Shells As Partial Replacement Of Coarse Aggregates In Asphalt Concrete; Leonardo Electronic Journal Of Practices And Technology (Lejpt); Academic Direct.Org/A09/145_152.Htm.

[8]. Rohith. N And J. Ranjitha(2013); A Study On The Marshall Stability Properties Of Warm Mix Asphalt Using A Zycotherm Chemical Additive; International Journal Of Engineering Research And Technology(IJERT); ISSN: 2278-0181, Vol. 2 Issue 7, July2013; IJERTV 21370455.Pdf 